\title{
Idiopathic camptocormia
}

INSERM

\section{Source}

INSERM. (1999). Orphanet: an online rare disease and orphan drug data base. Idiopathic camptocormia. ORPHA:1320

Idiopathic camptocormia is a postural disease characterized by an anterior flexion of the torso (during walking or standing) that resolves in the supine position and that is caused by weakness of the lumbar paraspinal muscles (spinal extensors), due to massive fatty infiltrations of posterior spinal muscles, without an identifiable etiology. 